\title{
The Interaction of a Baroclinic Mean Flow with Long Rossby Waves
}

\author{
A. Colin de Verdière \\ Laboratoire de Physique des Océans, Brest, France \\ R. TAILleuX \\ Climate System Analysis Group, University of Cape Town, Cape Town, South Africa
}

(Manuscript received 16 September 2003, in final form 1 September 2004)

\section{ABSTRACT}

The effect of a baroclinic mean flow on long oceanic Rossby waves is studied using a combination of analytical and numerical solutions of the eigenvalue problem. The effect is summarized by the value of the nondimensional number

$$
R^{2}=\beta \frac{N^{2} h}{f^{2} U_{z}}
$$

when the mean flow shear keeps a constant sign throughout the water column. Because previous studies have shown that no interaction occurs if the mean flow has the shape of the first unperturbed mode (the non-Doppler shift effect), an implicit assumption in the application of the present work to the real ocean is that the relative projections of the mean flow on the second and higher modes remain approximately constant. Because $R^{2}$ is large at low latitudes between $10^{\circ}$ and $30^{\circ}$ (the southern branches of subtropical gyres or the regions of surface westward shear), the phase speed of the first mode is very slightly decreased from the no mean flow standard theory case. Between $30^{\circ}$ and $40^{\circ}$ latitudes (the northern branches of the subtropical gyres or the regions of surface eastward shear), $R^{2}$ is $O(10)$ and the westward phase speed can increase significantly (up to a factor of 2). At still higher latitudes when $R^{2}$ is $O(1)$ a critical transition occurs below which no discrete Rossby waves are found that might explain the absence of observations of zonal propagations at latitudes higher than $50^{\circ}$. Our case studies, chosen to represent the top-trapped and constant-sign shear profiles of observed mean flows, all show the importance of three main effects on the value of the first baroclinic mode Rossby wave speed: 1) the meridional gradient of the quantity $N^{2} / f$ (where $N$ is the buoyancy frequency) rather than that of the potential vorticity $f N^{2} ; 2$ ) the curvature of the mean flow in the vertical direction, which appears particularly important to predict the sign of the phase speed correction to the no-mean-flow standard theory case: increase (decrease) of the westward phase speed when the surface-intensified mean flow is eastward (westward); and 3) a weighted vertical average of the mean flow velocity, acting as a Doppler-shift term, which is small in general but important to determine the precise value of the phase speed.

\section{Introduction}

The adjustment of the ocean to varying atmospheric forcing is carried out by Rossby waves whose properties have been widely studied. Although the interaction of such waves with mean flows lies at the heart of ocean circulation theories (e.g., Pedlosky 1996), it is always difficult to put the discussion on an observational basis given the difficulty of observing these slow, large-scale waves. The long-wave limit (for wavelengths large in comparison with the internal Rossby radius of defor-

Corresponding author address: Dr. A. Colin de Verdière, Laboratoire de Physique des Océans, UBO/UFR Sciences et Techniques, 6 avenue Le Gorgeu, CS 93837, Brest 29238 Cedex 3, France. E-mail: acolindv@univ-brest.fr mation) was given renewed attention when using Ocean Topography Experiment (TOPEX)/Poseidon satellite altimetry, Chelton and Schlax (1996) showed westward propagations of sea surface heights with wavelengths of several hundreds of kilometers and periods of several hundred days that fit into the range of scales of long baroclinic Rossby waves. Standard long Rossby wave theory, however, was judged unable to rationalize the observed "too fast" propagation speeds between $15^{\circ}$ and $50^{\circ}$ of latitude, say. At lower latitudes the long-wave hypothesis is certainly not appropriate and dispersion effects become important (Chang and Philander 1989). At higher latitudes the zonal propagations of large-scale anomalies are simply not observed. A complete review was carried out recently by Fu and Chelton (2000), which includes both the satellite obser- 
vations and the much earlier propagations of thermal signals observed for the most part in the North Pacific. Again the conclusion was that the standard theory underestimated the observed phase speeds of midlatitude upper thermal anomalies.

Given the order of magnitude of these observed phase speeds that become close to surface mean oceanic speeds as the latitude is increased, a wave-mean flow interaction has to be expected. The first solution that comes to mind is perhaps the boundary-trapped wave, which owes its existence to the presence of a horizontal (and of course vertical) temperature gradient. On the $f$ plane, this interesting wave, trapped at the upper boundary, does not propagate with the surface mean velocity but instead with the velocity at a Rossby height from the surface, the Rossby height being $\mathrm{fl}$ $N K_{H}$ (with $K_{H}$ the horizontal wavenumber; see Gill 1982 , p. 550). However for scales of interest large compared to the Rossby radius, the Rossby height exceeds the ocean depth and the solution does not apply readily. In this limit the upper wave interacts with a similar bottom wave to build up the Eady unstable wave. Furthermore, the large scale also implies the importance of the spherical shape of the earth. Schopp (1988) extended the long Rossby wave theory with a two-layer model to study the combined effects of a barotropic mean flow and nonlinearities whose origins lie in the finite displacement of the interface. However, far from western boundaries the barotropic circulations are too low to significantly influence the phase speed of the first mode. Killworth et al. (1997) extended the standard long Rossby wave theory to include a baroclinic mean flow. From the climatology of the World Ocean, they computed the geostrophic zonal flow and solved the eigenvalue problem locally over a flat bottom. Among all eigenvalues, they selected the one with the least value of the real part of the phase speed (i.e., the fastest westward wave propagation) and concluded that the presence of baroclinic mean flows was sufficient to account for the majority of satellite altimetry observed phase speeds. A different explanation by Tailleux and Mc Williams (2000, 2001) proposed that the factor of about 2 discrepancy between theory and observations was due to the fact that the waves did not feel the bottom of the ocean, the so-called pressure compensation hypothesis through which the wave pressure vanishes at the bottom. This effect is similar to the opening of one of the ends of a vibrating pipe so that the gravest mode fits one-quarter (instead of one-half) wavelength along the pipe. However the central question that remains with this theory is just the physical explanation for "opening the pipe." Although this hypothesis is difficult to accept at first sight, Rhines (1977) indicated that it emerges naturally when topographic slopes are so strong as to exceed the $\beta$ effect, while Samelson (1992) suggests this may also happen over rough topography. Killworth and Blundell (1999) used Wentzel-Kramers-Brillouin theory to study the effect a slowly varying topography on baroclinic rays and concluded that this effect, while important locally, is unimportant on average because it slows down and speeds up the waves alternatively. In spite of this, Killworth and Blundell $(2003 \mathrm{a}, \mathrm{b})$ found the topographic effect to improve the theory of Killworth et al. (1997) in which the phase speeds were still too low. As a possible explanation for this somewhat intriguing role of topography, it is important to realize that Killworth and Blundell (1999) did not systematically look in this case at the fastest wave present in the system, so their conclusion should be regarded with caution; see Tailleux (2003) for further discussion. In fact, a full understanding of topographic effects probably requires a theory valid for both the small scales as well as the large scales of the topography, given the above indications that small scales may also systematically speed up first baroclinic Rossby waves in the way described by the bottom pressure compensation theory of Tailleux and McWilliams (2001).

Although Killworth et al's (1997) inclusion of a baroclinic mean flow (and possibly of topography; see Killworth and Blundell 2003a,b) looks decisive, their global climatological calculation of phase speeds does not explain why the ocean shows such sensitivities. Dewar (1998) discussed the solutions of a three-layer quasigeostrophic model driven by a combination of steady and oscillatory forcing to show that the corrections to the standard theory were cyclonic, decreasing (increasing) the phase speeds at low (high) latitudes in the subtropical gyre, against the sense due to the Doppler shift associated with the anticyclonic circulation of the gyre. He attributed the sign of this peculiar effect to the vertical shear distribution of his steady flow and emphasized that three layers were the minimum to show this effect. A similar model was used by de Szoeke and Chelton (1999) to study the effect of a vanishing mean potential vorticity gradient in the middle layer, an assumption supported on several theoretical and observational grounds. They were able to demonstrate that their mean flow configuration led to an increase of the westward phase speeds in agreement with the Killworth et al. (1997) remark about the importance of secondmode structure in the mean flow. The 21/2-layer model was further analyzed by Liu (1999), who identified the eigenvalues for all combinations of the shear. He describes the first mode as an $N$ mode, a non-Doppler shift mode and the second mode as an $A$ mode, an advective mode as well as an unstable Green mode which is not our subject here. The $N(A)$ modes are weakly (strongly) mean shear dependent. Liu finds that the strongest wave speed enhancements are found in the case of a surface eastward flow ( $N$ mode) and for the case of a subsurface westward flow ( $A$ mode). He identifies a lack of wave speed enhancement for the $N$ mode in surface westward flows, which is in contrast with the results of Killworth et al.'s (1997) continuously stratified study. We also mention the different character of the studies by Spydell and Cessi (2003) and Ben 
Jelloul and Huck (2003), whose objectives are to compute the global modes of an ocean basin instead of the local modes as in previous studies. Applications to twolayer, idealized mean circulations have already been done, but further extension to more realistic situations have the drawbacks of requiring the full knowledge of the horizontal and vertical structure of a mean basin circulation, which solutions can only be provided from general circulation models with all the inherent difficulties of such an approach.

In the spirit of the above three-layer studies, the objective of the present work is to gain an improved understanding of the wave-mean flow interaction in the continuously stratified case through a combination of analytical and numerical solutions of idealized cases. We will show that, when the sign of the vertical shear of the mean flow is constant, the interaction is governed by the parameter

$$
R^{2}=\frac{\beta N^{2} h}{f^{2} U_{z}},
$$

which is the ratio of $\beta$ to its topographic equivalent induced by the slopes $f U_{z} / N_{2}$ of the mean density surfaces. Applying this result to the real ocean would require the implicit assumption that the relative projections of the mean flow on the second and higher baroclinic modes remain approximately constant from one region to the next since only these projections contribute to the modification of phase speed because of the so-called non-Doppler shift effect (e.g., Killworth et al. 1997; Dewar 1998; Liu 1999). This restriction is necessary because clearly the parameter $R^{2}$ alone cannot determine entirely the propagation characteristics of the baroclinic modes. Present observations suggest that the family of single-signed surface intensified mean shear is an important one to study: it will be our primary objective. Given the observations of westward propagation and the importance of dealing with nondispersive waves if the plane wave restriction is to be avoided, we restrict the mean flow to be purely zonal. Analytical results are first presented for cases of uniform stratification and linear shear (section 3 ) for which $R^{2}$ is constant throughout the ocean column, and then for a piecewise constant shear and buoyancy frequency (section 4) for which $R^{2}$ is piecewise constant over depth, being finite (infinite) in the upper (deep) ocean. Numerical solutions are given in section 5 for observed mean flow and stratification typical of midlatitudes. The analysis shows that a significant increase in westward phase speeds is limited to intermediate values of $R^{2}$ between 1 and 10 with the sign corrections as predicted earlier by Dewar (1998) and Liu (1999). Such values of $R^{2}$ correspond in the ocean to a rather narrow latitudinal window between, say, $30^{\circ}$ and $50^{\circ}$. The modal structures for buoyancy are very much top trapped so that long period variability, if interpreted as such free modes, is likely to be present only in the first few hundred meters near the surface. Section 6 is a discussion of the previous results in the light of an equation that gives the phase speed as depth integrals over the eigenfunctions. It is then possible to isolate the role of the mean flow curvature.

\section{Governing equations}

Conservation of buoyancy imply that the linearized perturbations of a mean zonal flow $U$ associated with mean buoyancy $B$ obey on the sphere:

$$
\frac{\partial b^{\prime}}{\partial t}+U \frac{\partial b^{\prime}}{a \cos \theta \partial \lambda}+\frac{v^{\prime}}{a} \frac{\partial B}{\partial \theta}+w^{\prime} \frac{\partial B}{\partial z}=0,
$$

where buoyancy $\left(-g \rho / \rho_{0}\right)$ and velocity have been written out as

$$
\begin{aligned}
& \mathbf{u}=\mathbf{u}^{\prime}+U \mathbf{i}(\mathbf{i} \text { zonal unit vector }) \text { and } \\
& b=b^{\prime}+B(\theta, z)
\end{aligned}
$$

$\lambda$ is longitude, $\theta$ is latitude, $z$ is increasing upward, and $a$ is the radius of the earth. Since both mean and perturbations obey the planetary geostrophic equations (geostrophy, hydrostatics, and incompressible fluid assumptions), we have

$$
f \mathbf{k} \times \mathbf{u}=-\nabla p / \rho_{0},
$$

whose curl gives

$$
\beta v=f w_{z} .
$$

Welander (1970) suggested the use of a new variable, $M$, such that

$$
p / \rho_{0}=M_{z} \text {. }
$$

With the use of (2) and (4), (3) can be integrated in the vertical direction (assuming that $w$ is zero at the bottom) to give

$$
w=\frac{\beta}{f^{2}} \frac{M_{\lambda}}{a \cos \theta} .
$$

Hydrostatic pressure allows one to relate $b$ and $M$ :

$$
b=M_{z z} .
$$

All dependent variables in (1) are now expressed in terms of the single variable $M$. After replacing $B_{z}$ by $N^{2}$ and after using the thermal wind to relate $U_{z}$ and $B_{\theta}$, (1) becomes the linearized form of Welander's $M$ equation:

$$
\begin{aligned}
& \left(\frac{\partial \cdots}{\partial t}+\frac{U}{a \cos \theta} \frac{\partial \cdots}{\partial \lambda}\right) M_{z z}^{\prime}-U_{z} \frac{M_{z \lambda}^{\prime}}{a \cos \theta}+\frac{\beta N^{2}}{f^{2}} \frac{M_{\lambda}}{a \cos \theta} \\
& \quad=0 .
\end{aligned}
$$

This equation has no meridional derivatives so that dependence in $\theta$ is parametric and waves, if any, are restricted to propagate zonally. Periodic solutions in longitude $e^{i m \lambda}$ at a given latitude $\theta_{0}$ are rewritten for sim- 
plicity as $e^{i k x}$, where $x=a \cos \theta_{0} \lambda$ and $k=m /\left(a \cos \theta_{0}\right)$. With $M^{\prime}=\operatorname{Re}\left[\phi(z) e^{i k(x-c t)}\right],(7)$ reduces to

$$
(U-c) \phi_{z z}-U_{z} \phi_{z}+\frac{\beta N^{2}}{f^{2}} \phi=0 .
$$

Since (8) is independent of $k$ (or $m$ ), the waves are nondispersive. Zero vertical velocity imposed at top and bottom requires

$$
\phi=0 \text { at } z=0 \text { and }-H .
$$

Relation (4) enforces the waves to be baroclinic, that is, to have vanishing vertical integrals of pressure and horizontal velocity. Equations (8) and (9) formulate the Killworth et al. (1997) eigenvalue problem for the eigenvalues $c$, which are determined up to a constant, the amount of barotropic zonal flow ${ }^{1}$ that can be freely added to the system. While the third term is the familiar Rossby wave term, the second, the mean shear, can be gauged against the third by scaling the $z$ dependence in perturbations with $h$ a vertical scale (which is of order $H$ for the gravest modes but may be much smaller than $H$ for the higher modes):

$$
\left(\frac{U-c}{U_{\eta}}\right) \phi_{\eta \eta}-\phi_{\eta}+R^{2} \phi=0,
$$

where $\eta=z / h$. Equation (10) reveals the central dimensionless number of this study:

$$
R^{2}=\frac{\beta N^{2} h}{f^{2} U_{z}},
$$

which is the ratio of the scale of the Rossby wave speed $\beta N^{2} h^{\prime 2} / f^{2}$ over the velocity variation of the mean flow $h U_{z}$ across the perturbation scale. It shows readily whether the mean flow variations are important. This parameter is also the ratio of $\beta$ to its topographic equivalent caused by the slope $f U_{z} / N^{2}$ of density surfaces. It is essentially the strong latitudinal dependence $\beta / f^{2}$ (as $\left.\cos \theta / \sin ^{2} \theta\right)$ that controls the value of $R^{2}$ in the ocean. Figure 1 shows that for typical large-scale circulation shears and first-mode structure $(h=H), R^{2}$ is indeed large at low latitudes to become order 1 at midlatitudes. It is then expected that the effect of the mean shear while negligible at low latitudes becomes stronger as the latitude increases. Since $R^{2}$ decreases with smaller $h$ values, one may also expect that the mean flow influence becomes progressively more important for the higher modes. Given its significance, this parameter is not new and has appeared in various other contexts. Here $R^{2}$ can be written as the ratio $h / h_{p}$, where $h_{p}\left(=f^{2} U_{z} / \beta N^{2}\right)$ has appeared in the literature as the vertical scale of the wind-driven ocean circulation (Young and Rhines 1982) and as the vertical scale of

\footnotetext{
${ }^{1}$ This effect amounts to a small Doppler shift because the barotropic velocity is usually much smaller than the first-mode Rossby wave speed.
}

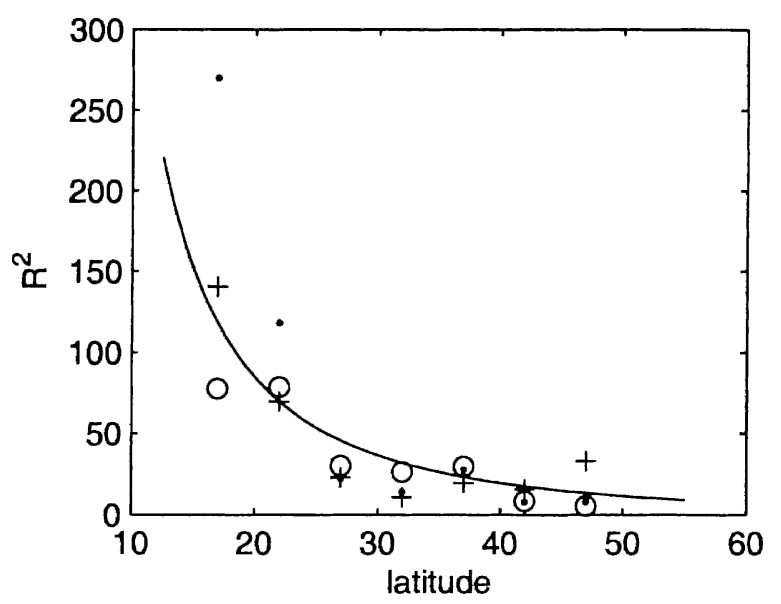

FIG. 1. The parameter $R^{2}=\beta N^{2} h / f^{2} U_{z}$ is shown as a function of latitude for $U_{z}=10^{-5} \mathrm{~s}^{-1}, h=4000 \mathrm{~m}$, and $N=2 \times 10^{-3} \mathrm{~s}^{-1}$. The open circles are surface values of $R^{2}$ for North Atlantic climatological data in the longitudinal band $45^{\circ}-36^{\circ} \mathrm{W}$ and $5^{\circ}$ latitudes ranges centered at $17^{\circ}, 22^{\circ}, 27^{\circ}, 32^{\circ}, 37^{\circ}, 42^{\circ}$, and $47^{\circ} \mathrm{N}$.

the most unstable wave in Charney's baroclinic instability problem (see Held 1978).

The eigenvalue problem, (8) and (9), has quite a variety of solutions. If $c$ is real and lies in the range of $U$ values, the point where $U=c$ is singular and the solution may not be well behaved. The spectrum of eigenvalues is said to be continuous as any $c$ in the interval $\left[U_{\min }, U_{\max }\right]$ may serve to construct a solution. If $c$ is real and lies outside this interval, discrete (isolated) eigenvalues may be found but (8) is not of SturmLiouville type, and so there is no proof of a complete spectrum of such discrete eigenvalues. There remains the possibility that $c$ be complex. Such possibilities were raised by Colin de Verdière (1986), Cavallini et al. 1988, and de Szoeke (1999). Colin de Verdière and Huck (1999) have argued that such instabilities are important in the western regimes of numerical ocean models forced by fixed fluxes of buoyancy at the surface. Given the focus on the steady waves in the ocean interior, these complex modes are not discussed any further in the present study.

\section{The constant shear case and constant $\boldsymbol{N}$ case}

For general stratification and background mean flow, the solution of the eigenvalue problem, (8) and (9), cannot usually be expressed in closed analytical form and, hence, must be computed numerically, as done previously by Killworth et al. (1997). With regards to physical understanding, however, the numerical approach has the obvious shortcomings of not providing much insight into the particular characteristics of the background mean flow and stratification that are important for the propagation of the first baroclinic mode. In this respect, analytical solutions are often of invaluable help, even when some of their features are unre- 
alistic. The constant shear case, discussed first, belongs to that category. Physically, the salient feature of this case is the vanishing of the curvature $U_{z z}$. This makes the westward and eastward cases symmetric and the first-mode phase speed $c$ slower than standard for all values of the shear. It follows that, to introduce an asymmetry between the westward and eastward cases and allow for a speed-up of the waves, a nonzero value of $U_{z z}$ is essential. Building on the constant shear case, we introduce in section 4 the model with two piecewise constant values of the shear (a delta function $U_{z z}$ ), which shows the same qualitative behavior as more realistic mean flows.

\section{a. Model formulation and adimensionalization}

To make the equations dimensionless, it is convenient to use as a velocity scale the speed of the first mode baroclinic Rossby waves in absence of shear. For a constant buoyancy frequency $N$ and ocean depth $H$, this speed is given by

$$
c_{0}=\frac{\beta N^{2} H^{2}}{f^{2} \pi^{2}} .
$$

The baroclinic mean flow is taken as $U=U_{z}(z+H / 2)$. Introducing the following dimensionless quantities:

$$
\begin{aligned}
U & =c_{0} \tilde{U}, \quad c=-c_{0} \gamma, \quad \eta=\frac{z}{H}, \quad U_{z}=\frac{c_{0}}{H} \tilde{U}_{\eta}, \\
R^{2} & =\frac{\pi^{2}}{\tilde{U}_{\eta}}, \quad \eta_{c}=\frac{1}{2}+\frac{\gamma R^{2}}{\pi^{2}}, \quad \text { and } \\
\gamma & =\frac{\pi^{2}}{R^{2}}\left(\eta_{c}-\frac{1}{2}\right),
\end{aligned}
$$

the following factor becomes, for a constant shear,

$$
\frac{U-c}{U_{z}}=H\left(\eta+\frac{1}{2}+\frac{\gamma}{\tilde{U}_{\eta}}\right)=H\left(\eta+\eta_{c}\right),
$$

allowing one to rewrite (10) as

$$
\left(\eta+\eta_{c}\right) \phi_{\eta \eta}-\phi_{\eta}+R^{2} \phi=0,
$$

where $R^{2}$ is a positive constant for the eastward shear case that we consider first.

\section{b. Reduction to a Schrödinger equation}

Giving attention to the discrete modes, $-\eta_{c}$ lies, then, outside the depth interval $[-1,0]$ : (i) If $\eta+\eta_{C}>$ 0 then $-\eta_{C}<-1$ (eigenvalues $c$ are negative), a new dependent variable $\psi$ is introduced:

$$
\phi=\left(\eta+\eta_{c}\right)^{1 / 2} \psi .
$$

Equation (11) becomes

$$
\psi_{\eta \eta}+\left[\frac{-3 / 4+R^{2}\left(\eta+\eta_{C}\right)}{\left(\eta+\eta_{C}\right)^{2}}\right] \psi=0
$$

There is a similarity in (12) with a Schrödinger equation in a potential

$$
V(\eta)=\frac{3 / 4-R^{2}\left(\eta+\eta_{C}\right)}{\left(\eta+\eta_{C}\right)^{2}}
$$

but the problem is more difficult because the eigenvalues " $\eta_{C}$ " do not appear linearly. The potential is a well and oscillatory solutions exist for $\eta$ above the turning point that occurs at $\eta=-\eta_{c}+(3 / 4) R^{-2}$. Discrete eigenvalues are to be expected.

(ii) If $\eta+\eta_{C}<0$, then $-\eta_{C}>0$ (eigenvalues $c$ are positive) and one introduces instead

$$
\phi=\left[-\left(\eta+\eta_{c}\right)\right]^{1 / 2} \psi,
$$

(11) becomes

$$
\psi_{\eta \eta}-\left[\frac{3 / 4-R^{2}\left(\eta+\eta_{C}\right)}{\left(\eta+\eta_{C}\right)^{2}}\right] \psi=0 .
$$

The coefficient of $\psi$ is now strictly negative and nonoscillatory solutions for $\psi$ will not allow for the existence of eigenvalues.

\section{c. Reduction to a Bessel equation of order 2}

Returning to case $\mathrm{i}$, there is another transformation of (11) that allows a closed form expression for the solutions in terms of Bessel functions if $\phi$ is transformed as

$$
\phi(\eta)=t^{2} H(t)
$$

where $t=2\left(\eta+\eta_{c}\right)^{1 / 2}$ when $\eta+\eta_{c}>0$. One obtains

$$
t^{2} \ddot{H}+t \dot{H}+\left(-4+R^{2} t^{2}\right) H=0,
$$

where the Bessel equation of order 2 is recognized. Its general solution, satisfying the upper boundary condition, takes the form

$$
H(t)=J_{2}\left(R t_{0}\right) Y_{2}(R t)-Y_{2}\left(R t_{0}\right) J_{2}(R t)
$$

(up to a multiplicative constant), where $t_{0}=2 \eta_{c}^{1 / 2}$, or, in terms of $\eta$,

$$
\begin{aligned}
\phi(\eta)= & \left(\eta+\eta_{c}\right)\left\{J_{2}\left(2 R \eta_{c}^{1 / 2}\right) Y_{2}\left[2 R\left(\eta+\eta_{c}\right)^{1 / 2}\right]\right. \\
& \left.-Y_{2}\left(2 R \eta_{c}^{1 / 2}\right) J_{2}\left[2 R\left(\eta+\eta_{c}\right)^{1 / 2}\right]\right\} .
\end{aligned}
$$

Applying the bottom boundary condition provides the eigenvalue equation that is the dispersion relation:

$$
J_{2}\left(R t_{0}\right) Y_{2}\left(R t_{b}\right)-Y_{2}\left(R t_{0}\right) J_{2}\left(R t_{b}\right)=0,
$$

where $R t_{0}$ and $R t_{b}$ are related to the dimensionless eigenvalue $\gamma$ by

$R t_{0}=2 R\left(\frac{1}{2}+\frac{\gamma R^{2}}{\pi^{2}}\right)^{1 / 2}$ and $R t_{b}=2 R\left(\frac{1}{2}-\frac{\gamma R^{2}}{\pi^{2}}\right)^{1 / 2}$.

Equation (14) represents an implicit equation for $\gamma$, which in general must be solved numerically. Note here that we only consider the case without a critical layer. 


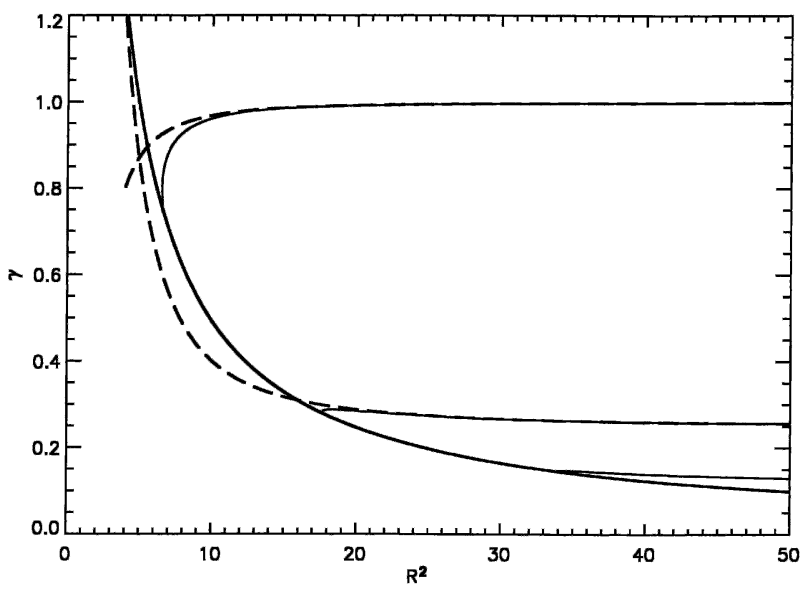

FIG. 2. Dispersion curves (thin lines) for the constant-shear case showing the phase speed $\gamma$ of the baroclinic modes, normalized by the speed of the zero mean flow first baroclinic mode, as a function of $R^{2}$. The thick solid line represents the critical curve $\gamma_{c}$ from (15), while the thick dashed lines represent the first-order approximation for the first two baroclinic modes, computed from (16). The points where the thin solid lines meet the thick solid critical curve are given in Table 1 and represent the places where the stable discrete modes cease to exist.

For an eastward shear, this imposes the constraint on the parameter $R t_{b}$ to remain positive, and hence

$$
\gamma>\frac{\pi^{2}}{2 R^{2}}
$$

This relation defines a critical curve $\gamma_{c}\left(R^{2}\right)=\pi^{2} / 2 R^{2}$ below which no stable discrete solution will be found (the possibility of unstable discrete modes and of continuous spectrum solutions is left for a further study). The dispersion curves in presence of shear flow are easily visualized by plotting the zero isovalue in a contour plot of (14) computed as a function of $\gamma$ and $R^{2}$. These dispersion curves are depicted in Fig. 2 onto which we also superimposed the critical curve $\gamma_{c}\left(R^{2}\right)=$ $\pi^{2} / 2 R^{2}$. As expected, discrete stable modes are found to the right of this critical curve. Only the first three baroclinic modes are visible with decreasing values of $\gamma$ on the figure. The first baroclinic mode appears slower than the standard phase speed for all values of $R^{2}$, while all the higher baroclinic modes are faster than their zero mean flow counterparts, as rationalized below.

Considering the westward-flow case, it can be shown that the problem is perfectly symmetric for the eastward and westward shear cases and that the eigenvalues are therefore identical. The eigenfunctions differ however, increasing (decreasing) toward the surface in the surface eastward (westward) mean flow cases.

\section{d. Asymptotics expression for large $R^{2}$}

To better understand the behavior of the modes, we computed asymptotic expressions of $\gamma$ for large $R^{2}$ using Mathematica. The computations, algebraically
TABLE 1. Summary for the first three modes.

\begin{tabular}{lccr}
\hline \hline & \multicolumn{3}{c}{$p$} \\
\cline { 2 - 4 } & 1 & 2 & 3 \\
\hline$R_{p}^{2}$ & 6.59 & 17.7 & 33.755 \\
$p^{2} \gamma_{p}$ & 0.75 & 1.114 & 1.316 \\
\hline
\end{tabular}

demanding, are not reproduced. The final result is given by

$$
\gamma=\frac{1}{p^{2}}\left[1+\frac{p^{2} \pi^{2}\left(p^{2} \pi^{2}-15\right)}{16 R^{4}}-\frac{135 p^{4} \pi^{4}}{256 R^{8}}+O\left(\frac{1}{R^{16}}\right)\right],
$$

where $p$ (the mode number) is an integer. To obtain this result, we made use of the following series expansion for the order-2 Bessel functions:

$$
J_{2}(x)=\sqrt{\frac{2}{\pi x}}\left[A(x) \cos \left(\frac{5 \pi}{4}-x\right)+B(x) \sin \left(\frac{5 \pi}{4}-x\right)\right]
$$

and

$$
Y_{2}(x)=\sqrt{\frac{2}{\pi x}}\left[B(x) \cos \left(\frac{5 \pi}{4}-x\right)-A(x) \sin \left(\frac{5 \pi}{4}-x\right)\right],
$$

where the functions $A$ and $B$ are given by

$$
\begin{aligned}
& A(x)=1-\frac{105}{128 x^{2}}+\frac{10395}{32768 x^{4}}+O\left(\frac{1}{x^{6}}\right) \text { and } \\
& B(x)=\frac{15}{8 x}+\frac{315}{1024 x^{3}}-\frac{135135}{262144 x^{5}}+O\left(\frac{1}{x^{7}}\right) .
\end{aligned}
$$

Equation (16) shows that the altered phase speed can be expressed as a regular series expansion in powers of $R^{4}$ and hence that, as expected, the result is independent of the sign of the shear. Furthermore, it is seen that the leading-order coefficient is negative for $p=1$, but positive for higher values of $p$. The formula therefore predicts a systematic slow down of the first baroclinic mode, but a systematic speed up of all higher baroclinic modes for small shear. These asymptotic results shown in Fig. 2 are excellent, even for rather small values of $R^{2}$.

Each dispersion curve meets the critical curve at a particular value of $R^{2}=R_{p}^{2}$. Because of the singular behavior of $Y_{2}$ near zero, the intersection points must be such that $J_{2}\left(R_{p} t_{0}\right)=0$, in addition to be on the above-mentioned critical curve. In this case there is a critical point just at the bottom $\left(\eta_{c}=1, t_{0}=2\right)$ so that the critical values of $R_{p}$ are given by $R_{p}=j_{2 p} / 2$, where $j_{2 p}$ are the zeros of the Bessel function $J_{2}\left(j_{2 p}=5.13562\right.$, 8.41724 , and 11.61984 obtained from Abramowitz and Stegun 1962). The result is summarized in Table 1 for the first three modes. It is readily seen from this table that the influence of a mean shear on Rossby modes increases markedly with the mode number and that even very small shears can suppress high Rossby modes. 
Let us summarize the results.

- When the shear is moderate, the mean flow correction slows down the first baroclinic mode irrespective of the sign of the shear but speeds up all the higher modes that are increasingly sensitive to shear.

- When the shear becomes larger a cut off appears when $R^{2}$ becomes $O(1)$. Below a critical value of $R^{2}$ (which is different for each mode), the stable discrete mode ceases to exist and leave the room for the continuous spectrum solutions and/or unstable discrete ones.

\section{A model with piecewise constant shear and $N$}

The symmetry between eastward and westward shears, as well as the systematic slowdown of the first baroclinic mode, are not observed for realistic mean flows, as is apparent from the results of Killworth et al. (1997), for instance. In this respect, a constant shear does not appear to approximate correctly the effect of a realistic background mean flow on the propagation of first mode baroclinic Rossby waves. On the other hand, we believe that it explains in an essentially correct way the disappearance of the baroclinic modes at low $R^{2}$ values (i.e., with increased shear or higher latitudes) for westward shears. Indeed, we show below that the eastward shear case allows the stable discrete modes to exist over a much broader range of shears in addition to show a systematic speedup of the first-baroclinic waves.

To improve upon the constant shear case treated previously, the simplest modification that comes to mind is to assume the constant shear to be restricted to the upper ocean, for $z$ between $-h$ and 0 , say, with a constant horizontal velocity in the deep ocean. With regard to the buoyancy frequency, the problem remains analytically tractable if two different values are assumed in the upper and lower ocean, but the analytical expressions for the phase speed become so complicated that no real physical insight can be gained; for this reason, we shall provide results only for the case where $N^{2}$ is constant throughout the water column, even though we shall formulate the problem for the case with two different values.

\section{a. Mathematical formulation of the problem}

As before, the problem is made dimensionless by using as a velocity scale the speed of the first baroclinic mode in absence of shear. For a piecewise buoyancy frequency, however, there is no simple expression for such speed. Nevertheless, it is possible to write it down formally as follows:

$$
c_{0}=\mu^{2} \frac{\beta N_{0}^{2} H^{2}}{f^{2}},
$$

where we know that $\mu$ reduces to $1 / \pi$ for a buoyancy frequency constant over the whole ocean depth. Accordingly, the parameter $R$ is now defined as

$$
R^{2}=\frac{1}{\mu^{2} \tilde{U}_{\eta}},
$$

where the shear is now different from zero only in the upper ocean. The dimensionless velocity and dimensionless buoyancy frequency $\nu$ (normalized by its value in the upper ocean) are chosen as

$$
\tilde{U}=\tilde{U}_{\eta}\left(\eta+\eta_{0}\right), \quad \nu=1, \quad \text { for } \quad-\delta \leq \eta \leq 0,
$$

and

$$
\tilde{U}=\tilde{U}_{b}, \quad \nu=\nu_{b}, \quad \text { for } \quad-1 \leq \eta \leq-\delta,
$$

where $\eta_{0}$ is determined by the constraint that the mean flow be purely baroclinic:

$$
\eta_{0}=\delta\left(1-\frac{\delta}{2}\right)
$$

which implies

$$
\tilde{U}_{b}=-\tilde{U}_{\eta} \frac{\delta^{2}}{2}=-\frac{\delta^{2}}{2 \mu^{2} R^{2}} .
$$

The eigenvalue problem can now be expressed for the upper ocean $(-h<z<0)$ as

$$
\begin{aligned}
\left(\eta+\eta_{c}\right) \phi_{\eta \eta}-\phi_{\eta}+R^{2} \phi & =0, \\
\eta_{c}=\eta_{0}+\frac{\gamma}{\tilde{U}_{\eta}} & =\eta_{0}+\gamma \mu^{2} R^{2},
\end{aligned}
$$

which is identical to the case studied in section 3 except that the expression for $\eta_{\mathrm{c}}$ is now different.

For the lower ocean $(-H<z<-h)$ :

$$
\left(\tilde{U}_{b}+\gamma\right) \phi_{\eta \eta}+\frac{\nu_{b}^{2}}{\mu^{2}} \phi=0 .
$$

As in the previous case, we only deal with the case with no critical level. To ensure that this is the case, the quantity $\tilde{U}+\gamma$ must be different from zero over the whole depth interval. Since $\gamma$ is positive by construction, this implies that $\tilde{U}_{b}+\gamma>0$ for eastward shear $\left(\tilde{U}_{\eta}>0\right)$ and that $\tilde{U}(\eta=0)+\gamma>0$ for westward shear $\left(\tilde{U}_{\sigma}<0\right)$. It can be verified that this takes the form

$$
\gamma>\frac{\delta^{2}}{2 \mu^{2} R^{2}} \text { for eastward shear }
$$

and

$$
\gamma>\frac{\delta(1-\delta / 2)}{\mu^{2} R_{0}^{2}} \text { for westward shear, }
$$

where $R_{0}^{2}=-R^{2}$. The previous constant-shear case is recovered for $\delta=1$, in which case we also have $\mu=1 / \pi$, and the condition becomes identical for eastward and westward shears, as expected.

\section{b. Dispersion relation and asymptotics}

It is straightforward, although algebraically tedious, to derive the dispersion relation for the piecewise con- 
stant shear and buoyancy frequency case. This dispersion is obtained by matching the solutions in the upper and lower part by assuming the solution and its first derivative to be continuous in the matching region. In distinction to the previous case, the symmetry between the westward and eastward cases is lost, and we need to treat the two cases separately. The results are summarized below:

For eastward shear, $\tilde{U}_{\eta}=1 / \mu^{2} R^{2}>0$. In this case, one shows that the dispersion relation can be written

$$
\Delta_{1} \sin \mathrm{X}-\nu_{b} \Delta_{2} \cos \mathrm{X}=0,
$$

where the quantities appearing in this expression are given by

$$
\begin{aligned}
\mathrm{X} & =\frac{\nu_{b}(1-\delta)}{\mu\left(\gamma+\tilde{U}_{b}\right)}, \\
\Delta_{1} & =Y_{2}\left(R t_{0}\right) J_{1}\left(R t_{b}\right)-J_{2}\left(R t_{0}\right) Y_{1}\left(R t_{b}\right), \\
\Delta_{2} & =Y_{2}\left(R t_{0}\right) J_{2}\left(R t_{b}\right)-J_{2}\left(R t_{0}\right) Y_{2}\left(R t_{b}\right), \\
R t_{0} & =2 R \eta_{c}^{1 / 2}=2 R^{2}\left[\gamma \mu^{2}+\frac{\delta}{R^{2}}\left(1-\frac{\delta}{2}\right)\right]^{1 / 2}, \quad \text { and } \\
R t_{b} & =2 R\left(\eta_{0}+\eta_{c}\right)^{1 / 2}=2 R^{2}\left[\gamma \mu^{2}-\frac{\delta^{2}}{2 R^{2}}\right]^{1 / 2} .
\end{aligned}
$$

For westward shear,

$$
\tilde{U}_{\eta}=-1 /\left(\mu^{2} R_{0}^{2}\right)<0, \quad R_{0}^{2}=-R^{2} .
$$

The dispersion relation for the westward shear can be expressed as

$$
\Delta_{1} \sin X+\nu_{b} \Delta_{2} \cos X=0
$$

where now

$$
R_{0} t_{0}=2 R_{0}\left(-\eta_{c}\right)^{1 / 2}=2 R_{0}^{2}\left[\gamma \mu^{2}-\frac{\delta}{R_{0}^{2}}\left(1-\frac{\delta}{2}\right)\right]^{1 / 2}
$$

and

$$
R_{0} t_{b}=2 R_{0}\left[-\left(\eta_{0}+\eta_{c}\right)\right]^{1 / 2}=2 R_{0}^{2}\left(\gamma \mu^{2}+\frac{\delta^{2}}{2 R_{0}^{2}}\right)^{1 / 2} .
$$

\section{c. Asymptotics for large $R^{2}$}

As in the constant shear case, it is possible to derive an asymptotic relation for $\gamma=c / c_{R}$ for large $R^{2}$ using Mathematica. The computations being algebraically demanding, only the final result is given:

$$
\gamma=\frac{1}{p^{2}}\left[1+\frac{3 \sin ^{2}(\delta p \pi)}{2 R^{2}}+\frac{a_{2}(\delta, p)}{R^{4}}+\cdots\right]
$$

or in terms of the dimensionless shear,

$$
\gamma=\frac{1}{p^{2}}\left[1+\frac{3 \sin ^{2}(\delta p \pi)}{2 \pi^{2}} \tilde{U}_{\eta}+\frac{a_{2}(\delta, p)}{\pi^{4}} \tilde{U}_{\eta}^{2}+\cdots\right],
$$

where $a_{2}(\delta, p)$ is analytically very complicated, and thus not written down here. This coefficient is shown for the first and second baroclinic modes in Fig. 3. As is seen, $a_{2}(\delta, p)$ is negative for all values of $\delta$ for the firstbaroclinic mode, but mostly positive for the second baroclinic mode. The expressions (19a) and (19b), which are valid for eastward as well as for westward shears, show that to leading order the wave speeds are increased by an eastward shear, but slowed down by a westward shear.

The dispersion curves [i.e., the zero isovalue of (18a) and (18b)], the critical curves (17a) and (17b), and the first-order asymptotic expressions (19a) are all depicted in Fig. 4 for the eastward and westward shear cases and for the two particular values of $\delta=0.25$ and $\delta=0.5$. Interestingly, it is seen that in the westward shear case the systematic slowdown of the first baroclinic mode and the vanishing of the higher baroclinic modes for low values of $R^{2}$ is essentially similar to that in the constant shear case discussed in section 3. Only the behavior of the higher baroclinic modes appear different, being no longer systematically accelerated by the mean flow. The eastward shear case, however, is markedly different from the westward shear case: not only do all baroclinic modes appear to be systematically accelerated compared to their zero mean flow counterparts, but their domain of existence appears also much broader with respect to the amplitude of the shear.

\section{d. Discussion}

We see first that the description of the mean flow effect on the phase speed can be made in terms of the dimensionless parameter $R^{2}$ computed for the upper part of the ocean, which answers the question of how this parameter is to be estimated for the real ocean. Second, it shows that the depth within which the maximum shear is confined plays a very important role and, third, it introduces an asymmetry between westward and eastward shears. There is indeed a broader domain of existence of the discrete modes in the case of the eastward shear than in the case of westward shear. It follows that in such regions of westward shear any variability might express itself more easily with other modes (unstable baroclinic modes and/or continuous spectrum solutions). Furthermore, this theory suggests the possibility to observe second (and perhaps higher) baroclinic modes. Cipollini et al. (1997) claim to have done so but their results have been questioned since then. As will be seen in the next section and in spite of an idealized nature, the model with a piecewise constant shear and buoyancy frequency bears a close relationship with more realistic cases.

\section{Observed vertical modes}

The previous analysis has suggested that the effect of the mean flow on phase speed is rather modest at low 

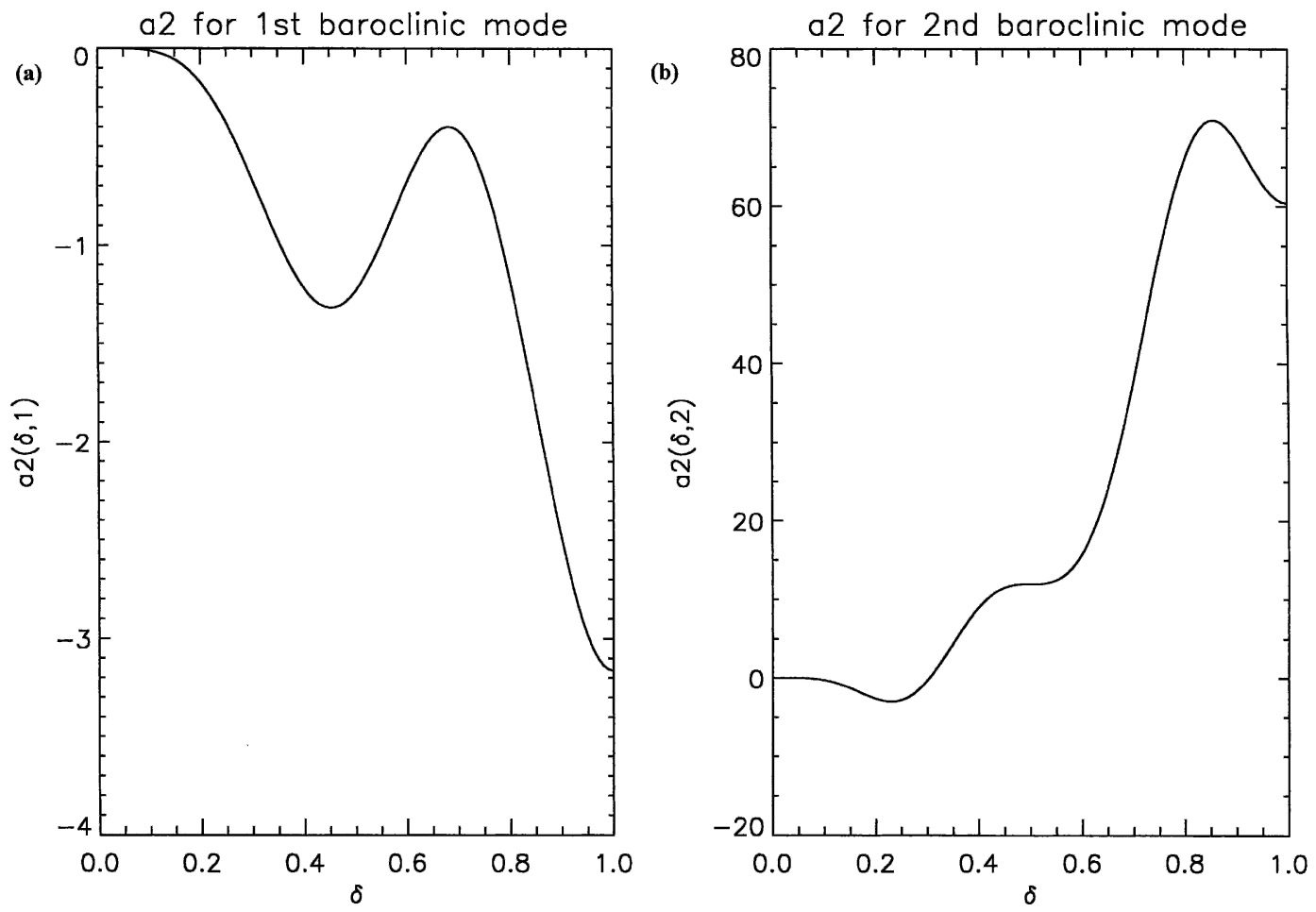

FIG. 3. The coefficient $a 2(\delta$, p) for (left) $p=1$ and (right) $p=2$, as a function of $\delta$.

latitudes and that the first mode may cease to exist at high latitudes. We give details to this picture by computing numerically the discrete modes of the observed circulation by solving (8) and (9). Killworth et al. (1997) have calculated in this way the westward phase speeds of the World Ocean in a pointwise manner, concluding that the inclusion of the mean flow was important to reconcile the factor of 2 (or so) difference between Rossby wave theory and the altimetry-derived phase speed (Chelton and Schlax 1996). With no intention to be exhaustive, we restrict our computations to the central parts of the North Atlantic excluding regions close to continental boundaries for which the assumption of a zonal mean flow would be a poor one. Brunt-Väisälä frequency and zonal velocity from respectively Reynaud's Atlantic hydrology database and Legrand's inverse analysis have been spatially averaged in boxes of $5^{\circ}$ latitude by $10^{\circ}$ longitude. The vertical profiles have been interpolated to 120 points uniformly spaced and the vertical average has been removed from the $U$ velocity (Fig. 5). The eigenvalue problem (8) and (9) has been solved using Matlab routine "eig" producing 120 modes and eigenvalues. These modes have been sorted out into three categories: discrete real modes whose $c$ lies outside the range of $U$ velocities, continuous real modes whose $c$ lies inside the range of $U$ velocities and complex modes with complex values of $c$ (which appear as complex conjugate pairs). The results that are shown hereafter belong only to the first category, the real discrete modes.

Figure 6 shows the phase speeds of the first mode for the North Atlantic as a function of latitude (every $5^{\circ}$ ) for the longitude interval $36^{\circ}-45^{\circ} \mathrm{W}$. The phase speeds decrease northward following roughly the $\beta / f^{2}$ latitudinal dependence of the parameter $R^{2}$. However the calculated speeds are above the $\beta / f^{2}$ curve by about 1 $\mathrm{cm} \mathrm{s}^{-1}$ between $25^{\circ}$ and $40^{\circ} \mathrm{N}$. Variations in $N$ and depth have been tested to explain that increase but it appears that only at $32^{\circ} \mathrm{N}$ can larger values of $N$ explain the departure. The rest presumably must come from mean flow effects. To illustrate these effects, Fig. 7 shows the phase speed of the first two modes normalized by the phase speed of the corresponding standard Rossby wave mode (calculated similarly with the observed stratification but with a $U=0$ condition). For latitudes less than $30^{\circ}$, the mean flow correction for the first mode is rather small and decreases the phase speed in spite of the westward direction of the surface mean flow. At latitudes higher than $30^{\circ} \mathrm{N}$ in the region of eastward surface flow, the correction becomes sizable (increasing the westward phase speeds) reaching a factor of 2 near $45^{\circ}-50^{\circ} \mathrm{N}$. What about the higher modes? First of all, only the second mode is usually present and all higher discrete modes have disappeared except at the lowest latitude $\left(15^{\circ}-20^{\circ} \mathrm{N}\right)$ or in regions where $U$ is quite small. When the second mode exists, the mean flow effects are increasingly important and the phase speed enhancement can reach a factor of 3 at the highest latitudes. The shape of the first mode is not very different from the shape of the standard $(U=0)$ Rossby mode. In both cases the vertical velocity has an 

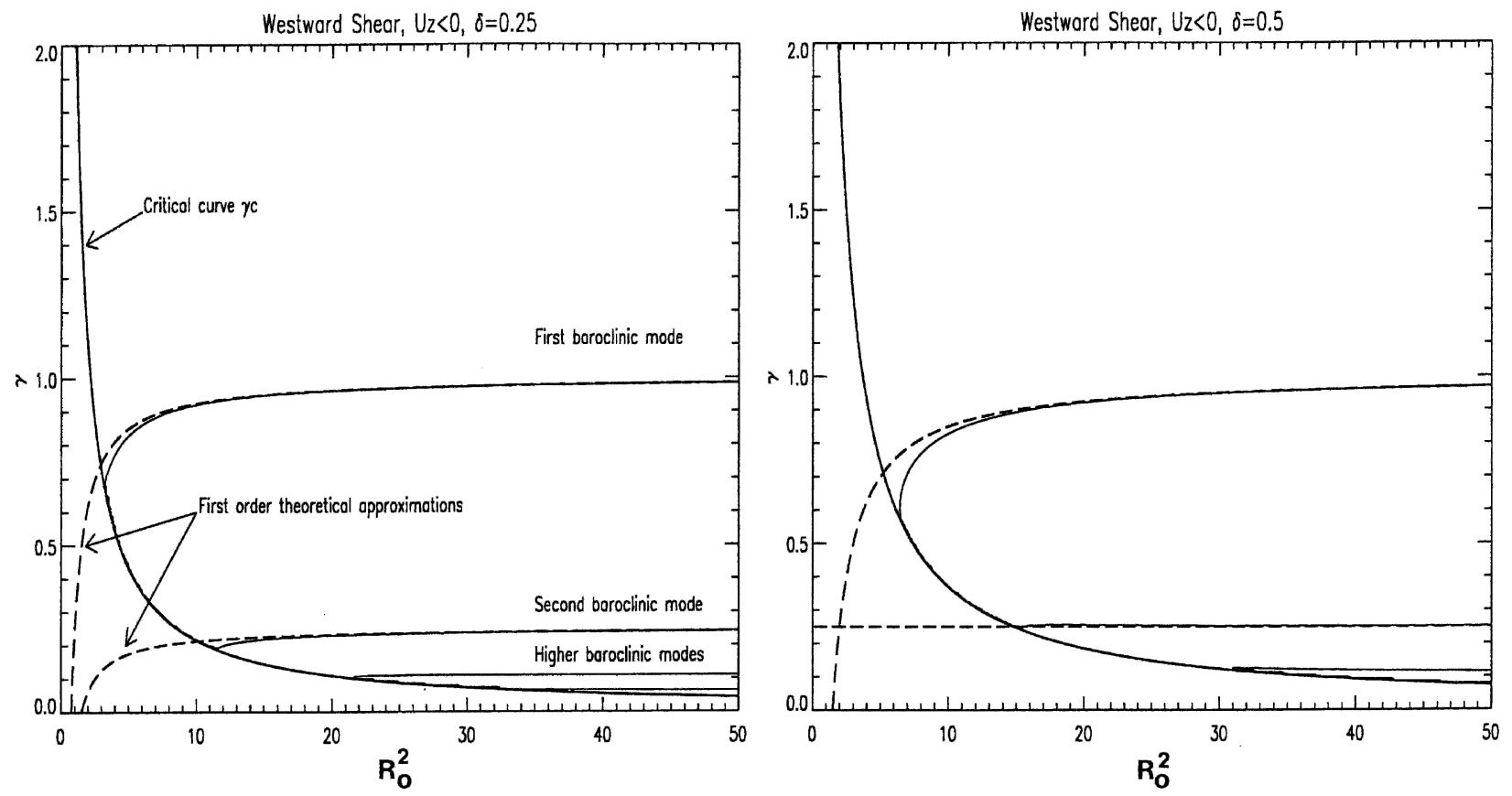

Eostward Shear, $U z>0, \delta=0.25$
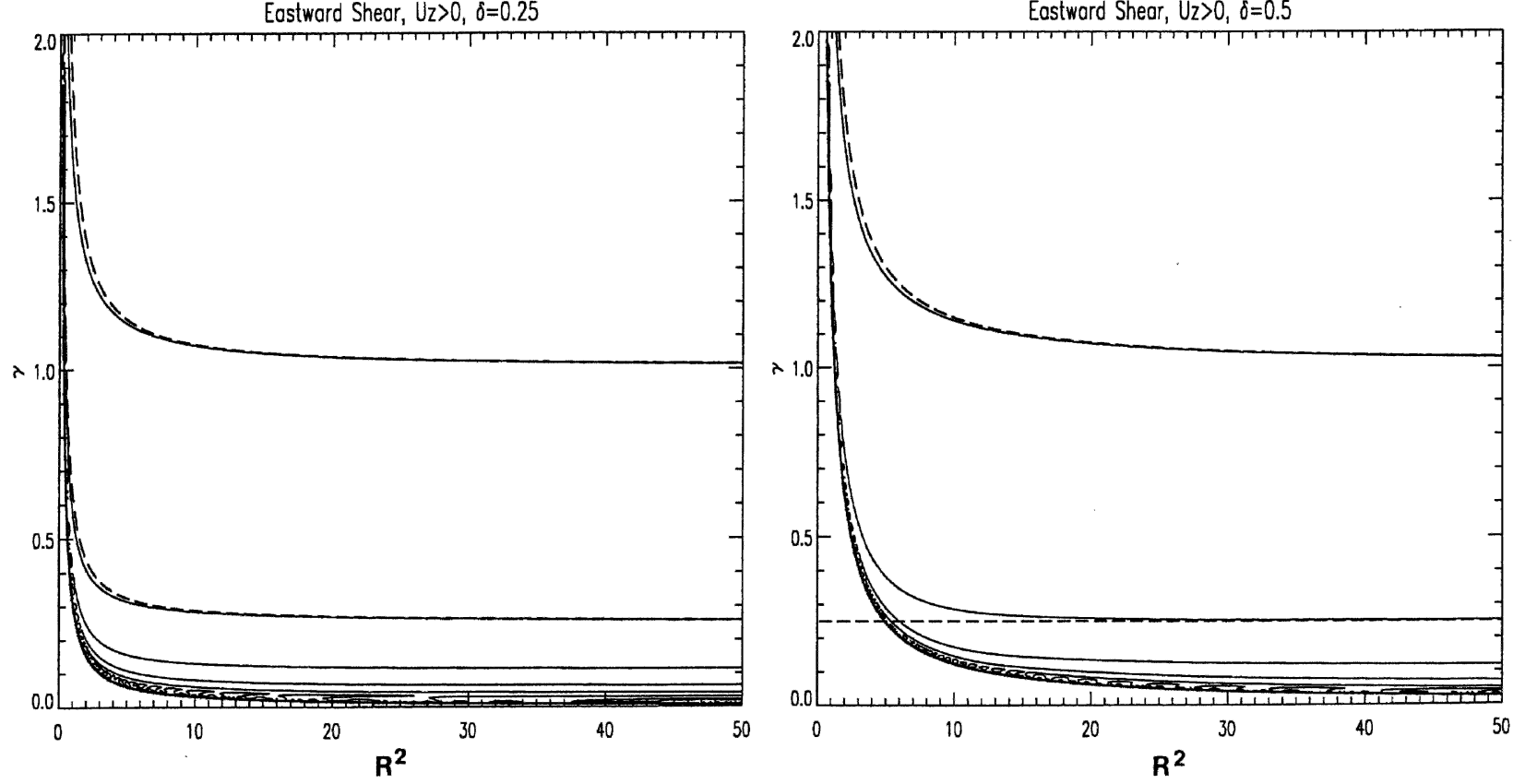

FIG. 4. The dispersion curves for the piecewise constant shear case, showing the phase speed of the baroclinic modes normalized by the zero mean flow first baroclinic mode phase speed, as a function of $R^{2}$, in the (top) westward shear case and (bottom) eastward shear case for the particular values of (left) $\delta=0.25$ and (right) $\delta=0.5$. Also represented are the critical curves (17a) and (17b) (thick solid line), as well as the first-order approximation computed from (19a) for the first two baroclinic modes (thick dashed lines). The main notations, common to all panels, are depicted in the top-left panel.

extremum around $1500 \mathrm{~m}$. Pressure $\left(\phi_{z}\right)$ is top trapped with a ratio of surface to deep amplitudes of about 10 . Only the modal shape for buoyancy (which is the second derivative of the vertical velocity) can depart somewhat from the standard case. The buoyancy is vanishingly small at depth and starts increasing toward the surface at a depth of about $500 \mathrm{~m}$. The increase is steep and can reach two orders of magnitude in the subtropical gyre with important consequences since temperature of the surface layers is the most commonly observed climatological parameter. If anything, the mean flow is observed to increase the top-trapped character 

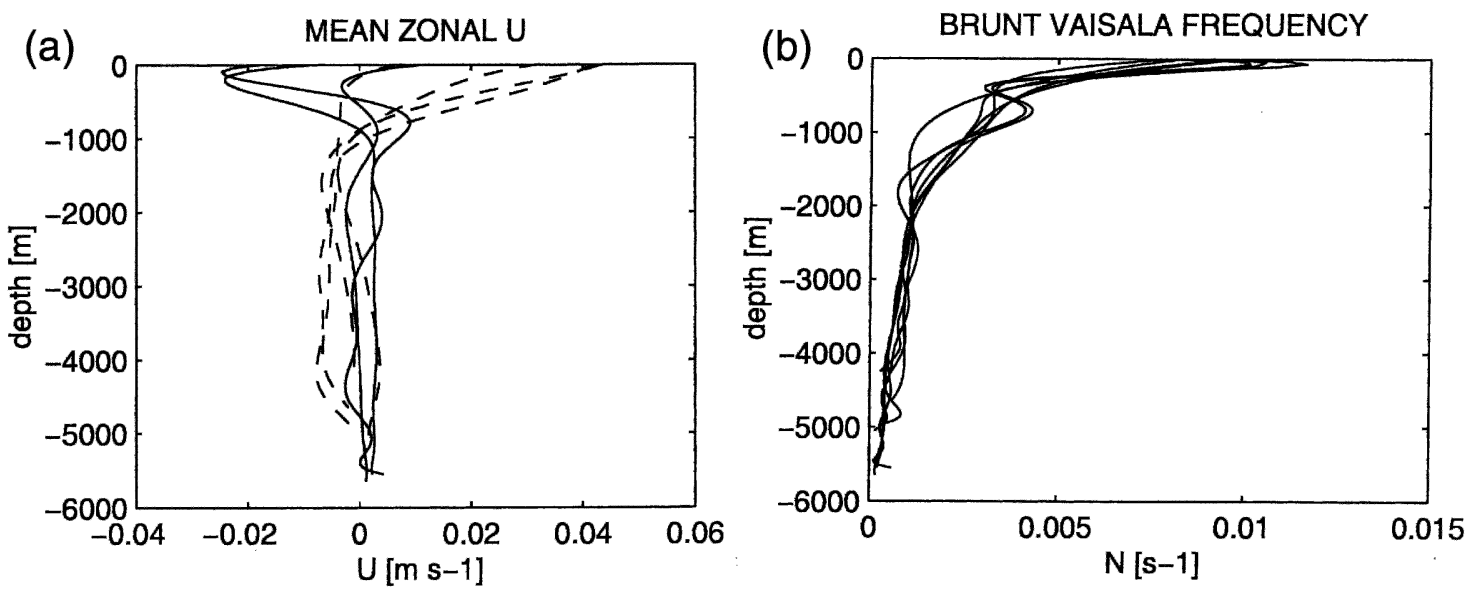

FIG. 5. The (a) mean zonal velocity and (b) Brunt-Väisälä frequency vertical profiles in the longitudinal band $45^{\circ}-36^{\circ} \mathrm{W}$ and $5^{\circ}$ latitude ranges centered at $17^{\circ}, 22^{\circ}, 27^{\circ}, 32^{\circ}, 37^{\circ}, 42^{\circ}$, and $47^{\circ} \mathrm{N}$. Solid (dashed) lines in (a) are from latitude bands lower (higher) than $30^{\circ} \mathrm{N}$.

of these buoyancy waves that may play an important role in long-term ocean variability.

To find out the sensitivity of these modes to the fundamental parameter $R^{2}$, it is necessary to explore variations of the mean shear. This analysis has been made for analytical (exponential) profiles that describe, albeit very approximately, the climatological vertical structure of $U$ and $N$. The mean flow shear is relatively constant in the main thermocline between 200 and 500 $\mathrm{m}$, but can vary rapidly near the surface. The shear is usually small at depth but small-scale structure often appears whose origin may come from insufficient data averaging and vertical interpolation. With the objective to represent only the large shear of the thermocline and the weak shear at larger depth, exponential profiles of

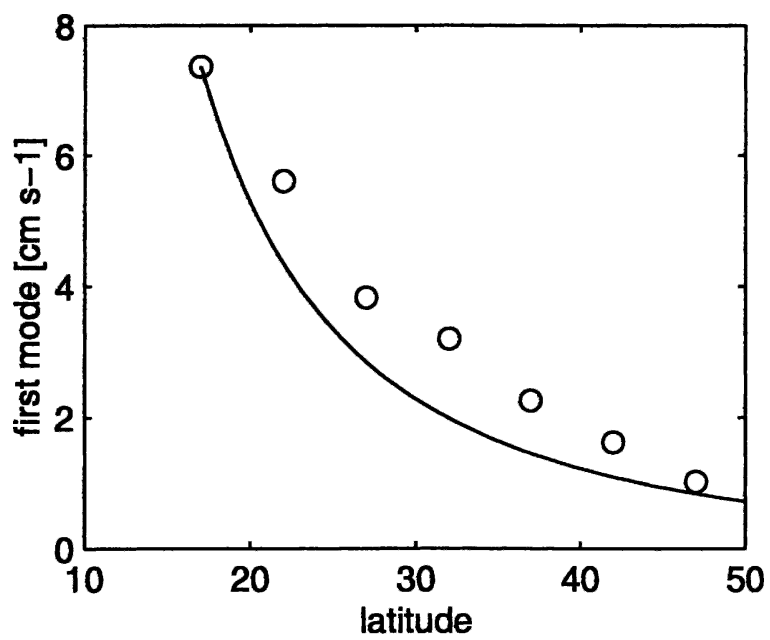

FIG. 6. The first-mode phase speed for the seven latitude bands defined in Fig. 5. The solid curve gives the $\cos \theta / \sin ^{2} \theta$ variation in latitude, which seems to explain most of the variation in phase speed.
$U$ and $N$ have been chosen, respectively, as $U_{1} e^{a z}$ and $N_{0}+N_{1} e^{a z}$ (with $a H=8$ and removing the vertical average of $U$ ). Of course this choice does not exhaust the possibilities: at a number of locations the mean flow presents one or two extrema in the first $1000 \mathrm{~m}$, which seem robust, and such situations will require separate analysis. The first discrete mode has been computed numerically for various $U_{1}$ keeping constant the BruntVäisälä frequency profile $\left(N_{0}=7.8 \times 10^{-3}, N_{1}=7.72\right.$ $\left.\times 10^{-4}\right)$, depth $(4000 \mathrm{~m})$, and latitude $\left(32^{\circ} \mathrm{N}\right)$. Both the case of eastward and westward shear have been considered. The first-mode eigenvalue scaled by the corresponding standard Rossby wave speed is shown in Fig.

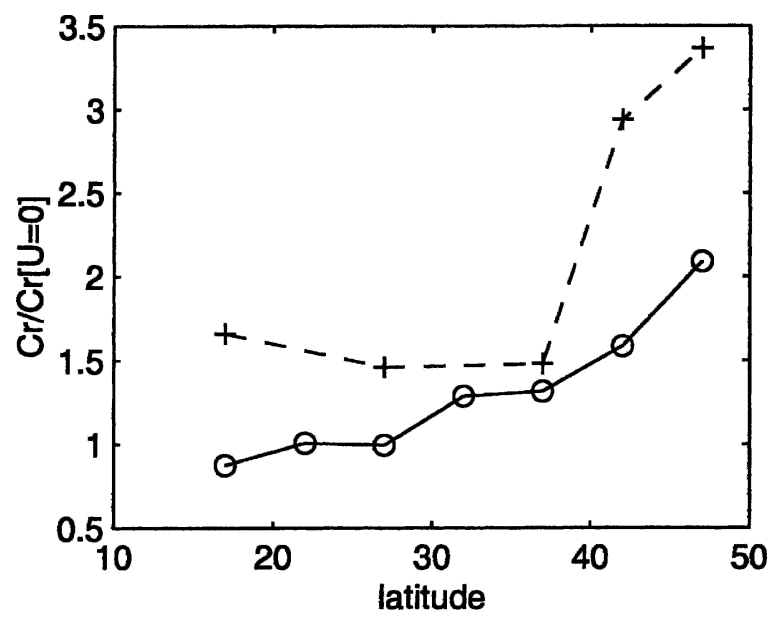

FIG. 7. The ratio of the first- $(\mathrm{o})$ and second- $(+)$ mode phase speed to the corresponding Rossby wave speed ( $U=0$ case). The effect of the mean flow on the first mode appears to be limited to the highest latitude band $\left(42^{\circ}\right.$ and $\left.47^{\circ} \mathrm{N}\right)$. The effect, which is stronger for the second mode (when it exists), amounts to a $50 \%$ increase at low latitudes and up to a factor of 3 for the highest latitudes. 

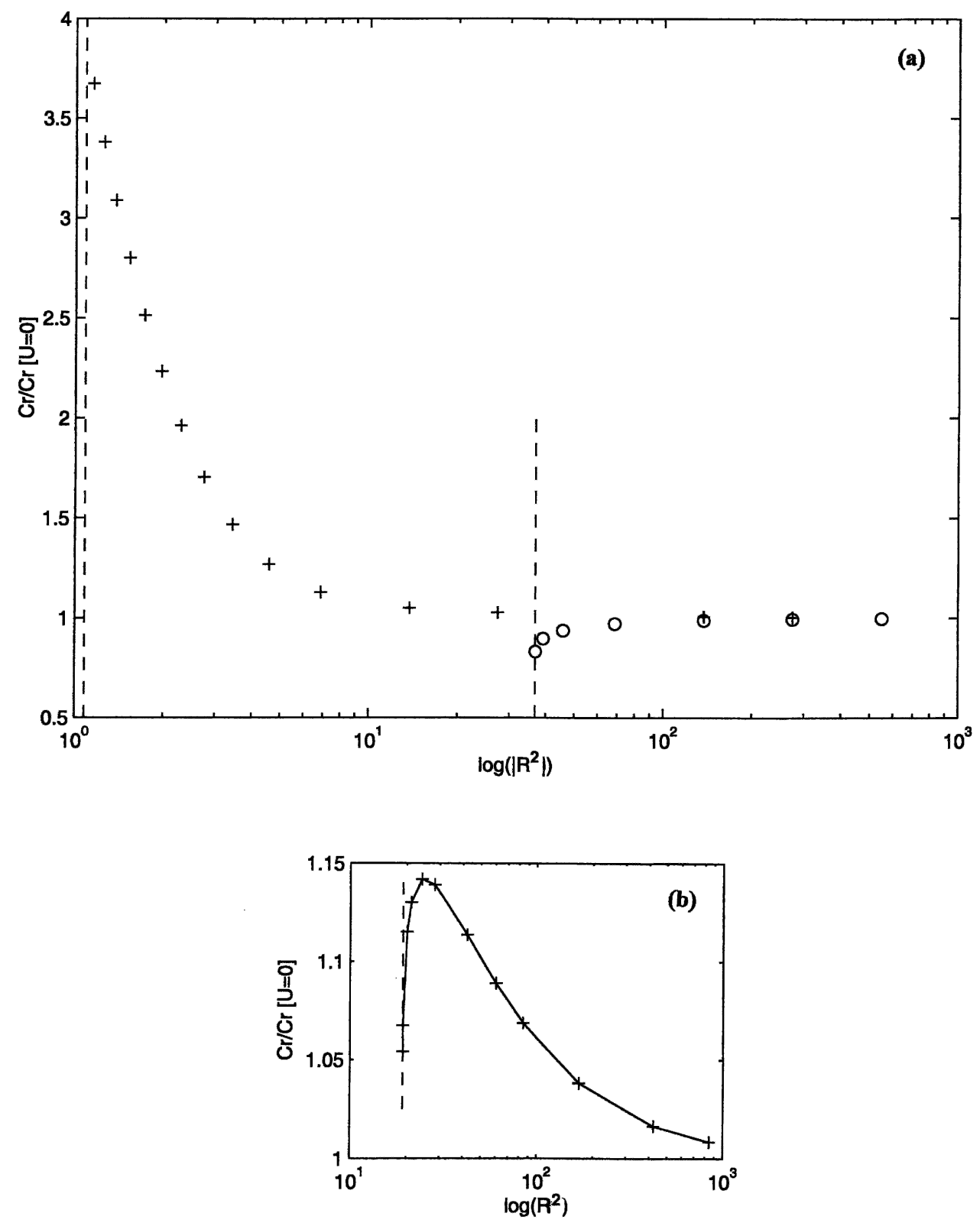

FIG. 8. Illustration of the first-mode phase speed for models of mean flow and stratification as a function of the parameter $R^{2}$ (evaluated at the surface). Variation of $R^{2}$ is produced by varying the surface amplitude of the mean flow keeping constant the vertical profile. The phase speed is normalized by that of the zero mean flow case. (a) A subtropical case $\left(30^{\circ} \mathrm{N}\right)$ for exponential stratification and exponential eastward (+) and westward (o) current. (b) An Antarctic circumpolar case $\left(50^{\circ} \mathrm{S}\right)$ with vertical profiles of mean flow and stratification adapted to the ACC. The climatology gives surface values of $R^{2} \approx 4$ well below the critical $R^{2}$.

8a against the nondimensional parameter $R^{2}$ estimated at the surface (with such exponential profiles of $U$ and $N$, the parameter $R^{2}$ varies little from the surface to $2000 \mathrm{~m}$ but increases markedly near the bottom). The eastward shear always increases the westward phase speed, by very small amounts for $R^{2}$ larger than 10 but up to a factor of 3 when $R^{2}$ approaches 1 . A cutoff is obtained for the existence of the first mode at a critical
$R^{2} \sim 1.14$. For westward shears, the westward phase speeds are now decreased in comparison with the standard Rossby wave speed. The decrease is small for $R^{2}$ larger than 100 , increasing to about $20 \%$ when the critical value of $R^{2} \sim 38.2$ is approached. There is a strong qualitative agreement between the results of the exponential cases and of the piecewise constant shear explored previously. Both cases share the small phase 
speed decrease and high cutoff values for $R^{2}$ when the shear is westward and the stronger phase speed increase and low cutoff values for $R^{2}$ when the shear is eastward.

Where do the North Atlantic observations fit on that sensitivity plot? We have not attempted to report the data points of Fig. 7 because of the arbitrariness in attempting to select values of $R^{2}$ when it is obviously not constant, even in the main thermocline. Near surface values of $R^{2}$ at latitudes less than $30^{\circ} \mathrm{N}$ for the westward surface mean flow case range from 10 to 200 , and Fig. 8a shows that very small reductions in westward phase speed are to be expected for the southern branch of the subtropical gyre. At latitudes higher than $30^{\circ} \mathrm{N}$ surface mean flow becomes eastward with $R^{2}$ ranging from 5 to 25 , and so a factor of 2 enhancement in phase speed is expected. At latitudes higher than $50^{\circ} \mathrm{N}$, the first mode ceases to exist if $R^{2}$ is less than one. Given the crudeness of the exponential model, we can only say that the sensitivities shown by the model agree qualitatively well with the observations in Fig. 7. Such sensitivities are consistent with Dewar (1998) who concluded from a completely different approach that the phase speeds corrections were opposed to the anticyclonic sense of the gyre.

The question of the presence of discrete modes in the Antarctic Circumpolar Current (ACC) is worth discussing briefly. The present theory is obviously more appropriate in the geometry of the ACC, and the results are obtained with appropriate fits of $U$ and $N$ to meridionally averaged quantities using the complete data from the Ajax section assumed representative enough of the ACC. Variations of $R^{2}$ are obtained similarly by varying the amplitude of the surface flow keeping constant all other parameters. The velocity profile is that of a broad eastward shear while the $N^{2}$ profile is more trapped to the surface. The eigenvalue diagram for the first mode in Fig. $8 \mathrm{~b}$ is somewhat similar to the previous eastward shear case with $10 \%-20 \%$ increase in westward phase speed but with an important difference. The critical value of $R^{2}$ below which no discrete modes are present is higher $\left(R^{2} \sim 19\right)$. When observed meridionally averaged values of the shear in the ACC are used, the value of $R^{2} \approx 4$ so obtained is lower than critical, and so no discrete long Rossby modes are to be expected in the region.

\section{Integral relations}

Integral relations are useful to shed some light on the links between the phase speed of the waves and their vertical modal structure. Since such relations are not unique however, the choice of a particular relation is often motivated by the need to illustrate a particular point. For instance, Killworth et al. (1997) derived an integral relation aimed at emphasizing that the perturbed phase speed is mainly due to the difference between two large terms, namely between the Doppler shift effect due to the mean flow and the modification of the background potential vorticity gradient; in that case, the integral relation is the best that can be derived to illustrate the non-Doppler shift effect that arises when the mean flow projects entirely on the first baroclinic mode because, when this is so, the two large terms cancel exactly. In this paper, we derive an integral relation whose main purpose is to show that the natural quantity affecting the phase speed is not the meridional gradient of the potential vorticity $f N^{2}$, as one would expect from the fact that the latter is a $3 \mathrm{D}$ materially conserved quantities along particle trajectories, but of the quantity $N^{2} / f$. The relation allows us to illustrate the role of $U$ and of its curvature $U_{z z}$ in a different way. To see this, let us multiply (8) by $\phi^{*}$ (complex conjugate) and add to the result the product of $\phi$ with the complex conjugate of (8). The expression is integrated with respect to $z$ over the depth interval and after several integrations by parts we obtain

$$
C_{r}=\frac{\int_{-H}^{0} U\left|\phi_{z}\right|^{2} d z-\int_{-H}^{0}\left(\frac{\beta N^{2}}{f^{2}}+U_{z z}\right)|\phi|^{2} d z}{\int_{-H}^{0}\left|\phi_{z}\right|^{2} d z} .
$$

The first term is akin to a Doppler shift by the mean flow, while the third shows directly the effect of its curvature. The weighting by the eigenfunctions is instructive: because the weighting of the Doppler shift term implies the first derivative of the eigenfunction, it is expected that the advective nature of the mode grows with the vertical structure of the mode at the expense of the size of third term, a result fully consistent with our previous calculations and which extends the 21/2-layer conclusion of Liu (1999) to the continuously stratified case. Consider now the case of positive curvature, that is, a near-surface intensified eastward mean flow. This $U_{z z}$ contribution appears analogous to a net increase of the stratification thereby increasing the westward phase speed. This is countered by the first term, which is certainly positive when the top-trapped character of the eigenfunctions is taken into account. The reverse is true for a near-surface westward mean flow where the curvature lowers the stratification. The expression helps to rationalize the behaviors of the first mode phase speeds for the top-trapped mean flows (piecewise constant shear and exponential profiles) examined in sections 4 and 5: we observe that in either cases the curvature effect dominates over that of the Doppler shift. Grouping together the second and third terms in (20), the gradient of the large-scale potential vorticity (pv) $Q=$ $f N^{2}$ (where $N^{2}=B_{z}$ ) would have been expected to emerge to control the phase speed, but this is not the case. The meridional gradient of $Q$ is

$$
\frac{\partial Q}{\partial y}=\beta N^{2}+f B_{z y}=\beta N^{2}-f^{2} U_{z z}
$$


after use of the thermal wind $\left(f U_{z}=-B_{y}\right)$. Although the mean pv gradient has been found so important for the instabilities (i.e., the imaginary parts of the phase speed), it does not appear to control the real part of the phase speed, and it is instead the meridional gradient of the quantity $N^{2} / f$, which appears when the second and third term in (20) are summed up. Suppose now (R. S. Schopp 2004, personal communication) that the mean flow is such that the gradient of potential vorticity vanishes, then the gradient of $N^{2} / f$ doubles, leading to an increase in westward first-mode phase speeds, which is to be compared with the $56 \%$ increase calculated by de Szoeke and Chelton (1999) when discussing the case of a vanishing pv mean flow. These comments, which are of a qualitative nature, are not meant to say that the first Doppler shift term is unimportant but that it does not govern the sign of the corrections to the first-mode phase speed for the family of mean flows profiles considered herein.

\section{Conclusions}

At low latitudes $\left(R^{2} \gg 1\right)$ and when the mean flow is westward (at the surface in the southern branches of the subtropical gyres), the effect of the mean flow is small and acts to decrease the standard phase speed. It is progressively much more important for the higher modes (usually no discrete modes higher than the first three are found). When the latitude increases, $R^{2}$ decreases and the mean flow effect increases. Based on exponential profiles of zonal flow and stratification, the phase speed variations for surface eastward flows between $30^{\circ}$ and $50^{\circ} \mathrm{N}$ (the northern branches of the subtropical gyres) can show an increase by a factor of 2 to 3 when $R^{2}$ is between 1 and 5. Only in this window is the mean flow contribution sizeable. At even higher latitudes (larger than $50^{\circ} \mathrm{N}$ ), when $R^{2}$ is less than values around one (eastward flow case), the first discrete mode ceases to exist altogether. The case of the ACC appears to be in this regime. For higher modes, the analytical examples constructed from linear shears show that the critical cutoff occurs at still higher values of $R^{2}$, that is, implying lower latitudes or weaker shears. Outside of these trapping regions, only the continuous modes are present. Because they have phase speeds in the range of the baroclinic mean velocities, they exhibit singularities for the buoyancy perturbations at the critical layer and cannot exist as such. They can become part of a physical picture only with the addition of instability (complex $c$ ), transience, and (or) mixing but cannot be part of a steady-state, conservative wave theory.

These numerical results can be reproduced qualitatively with a simple analytical model with piecewise constant shear, which shows the importance of the curvature of the mean flow $U_{z z}$. The existence of cutoffs for $R^{2}$ values of order one, the perhaps surprising effect of westward (respectively, eastward) shear at the surface which decrease (respectively, increase) the westward speeds of the standard theory, are all reproduced with the piecewise constant shear case. We have shown how this behavior may be discussed with the help of an integral relation for the phase speed (given in section $6)$, which shows that the mean flow curvature plays the role of a stratification (positive curvature increasing the stratification) and that this effect dominates over that of advection (the Doppler shift term). Surprisingly, it is not the gradient of potential vorticity that appears in the expression for the phase speed but that of the quantity $N^{2} / f$. We stress that the importance of the curvature term in (20) that we report has been obtained under the assumptions of constant sign shear profiles, with large shear in the thermocline and small shear below. Our conclusions agree qualitatively with Dewar's (1998) and Liu's (1999) low-resolution (three layer) work. There are indeed exceptions to the behavior found in our study. First, Killworth et al. (1997) draw attention to the noninteraction of wave and mean flow when $U$ has the shape of a standard $(U=0)$ baroclinic mode. As was pointed out to us by one reviewer, this same study gives examples (their Fig. 14) where the Doppler shift term is as important as the curvature term. In these examples, the mean flow profiles have more vertical structure with several internal extrema and fall out of the top-trapped, constant sign shear cases studied herein.

In their review of the observations, $\mathrm{Fu}$ and Chelton (2000) consider that within $15^{\circ}$ and $30^{\circ}$ latitude, the standard theory without a mean flow can be judged adequate to explain most of the observations. This is consistent with the present results: the large values of $R^{2}$ that are found at those latitudes imply indeed very small corrections to the standard theory. This is also consistent with the studies of Sturges and Hong (1995; Sturges et al. 1998), who have found the standard theory adequate to reproduce the sea level observations from a long-wave model forced by wind stress curl variability at decadal periods. It is at latitudes between $30^{\circ}$ and $50^{\circ}$ that the increase in westward phase speeds and a call to the baroclinic mean flow theory is justified to rationalize the observations. The present study shows that, when the mean flow effect is such that the parameter $R^{2}$ is $O(1)$, we are in the neighborhood of the cut off for the existence of the first mode. We conjecture that the absence of observed zonal propagation at high latitudes may be due to this effect. This is an alternative to the view that the absence of Rossby waves might be due to the existence of critical latitudes, which appear when dispersive effects are kept into account, poleward of which Rossby waves are evanescent (Gill 1982).

Although Killworth et al. (1997) find an increase (albeit a small one) in westward phase speeds at low latitudes, the present study, as that of Dewar (1998), shows a decrease in the regions of westward mean flow 
(the southern branch of the subtropical gyre). In their calculations of phase speeds for the World Ocean, two criteria were used by Killworth et al. (1997) to select phase speeds at each location: the first (which was finally chosen) was a criterion of minimum value of the real part of the phase speed, while the second was a criterion of smoothness in the vertical. In the words of these authors, "These two solutions were identical only in about half the locations, with the smooth solution preferentially having a very weak east-west phase speed dependence." Automating the choice of eigenvalues is far from a trivial task, however. As recalled earlier, continuous spectrum solutions are not smooth (being singular at the critical level) and complex eigenvalues are common as well: it is possible that their criterion selected eigenvalues pertaining to these latter categories instead of the discrete spectrum one.

A natural extension of the present work is to continue the examination of "idealized case studies" to include richer mean flow structures beyond the monotonic profiles studied herein and explore the predictive power of the integral relation (20). This same approach could also be applied to the combined mean flowtopography problem to find out where and if Tailleux and McWilliams' (2001) deep pressure compensation can explain some of the observations.

Acknowledgments. We are very much indebted to the reviewers who provided a thorough and informative review of a first draft of this paper. We wish to thank Pascal Legrand and Thierry Reynaud for providing, respectively, the North Atlantic velocity and BruntVäisälä frequency used in this study, and Richard Schopp for useful comments.

\section{REFERENCES}

Abramowitz, M., and I. A. Stegun, 1962: Handbook of Mathematical Functions. Dover, 1046 pp.

Ben Jelloul, M., and T. Huck, 2003: Basin-mode interactions and selection by the mean flow in a reduced-gravity quasigeostrophic model. J. Phys. Oceanogr., 33, 2320-2332.

Cavallini, F., F. Crisciani, and R. Mosetti, 1988: Bounds on the eigenvalues of the planetary-scale baroclinic instability problems. Dyn. Atmos. Ocean., 12, 71-80.

Chang, P., and S. G. H. Philander, 1989: Rossby wave packets in baroclinic mean currents. Deep-Sea Res., 36A, 17-37.

Chelton, D. B., and M. G. Schlax, 1996: Global observations of oceanic Rossby waves. Science, 272, 234-238.

Cipollini, P., D. Cromwell, M. S. Jones, G. D. Quartly, and P. G. Challenor, 1997: Concurrent altimeter and infrared observations of Rossby wave propagation near $34^{\circ} \mathrm{N}$ in the Northeast Atlantic. Geophys. Res. Lett., 24, 889-892.

Colin de Verdière, A., 1986: On mean flow instabilities within the planetary geostrophic equations. J. Phys. Oceanogr., 16, 1985-1990.

-, and T. Huck, 1999: Baroclinic instability; An oceanic wavemaker for interdecadal variability. J. Phys. Oceanogr., 29, 893-910. de Szoeke, R., 1999: An improved bound for the complex phase speed of baroclinic instability. J. Phys. Oceanogr., 29, 83-90. - and D. B. Chelton, 1999: The modification of long planetary waves by homogeneous potential vorticity layers. J. Phys. Oceanogr., 29, 500-511.

Dewar, W. K., 1998: On "too fast" baroclinic planetary waves in the general circulation. J. Phys. Oceanogr., 29, 500-511.

Fu, L.-L., and D. B. Chelton, 2000: Large-scale ocean circulation. Satellite Altimetry and Earth Sciences, L. L. Fu and A. Cazenave, Eds., International Geophysics Series, Vol. 69, Academic Press, 133-169.

Gill, A. E., 1982: Atmosphere-Ocean Dynamics. Academic Press, $662 \mathrm{pp}$.

Held, I., 1978: The vertical scale of an unstable baroclinic wave and its importance for eddy heat flux parameterizations. $J$. Atmos. Sci., 35, 572-576.

Killworth, P., and J. R. Blundell, 1999: The effect of bottom topography on the speed of long extratropical planetary waves. J. Phys. Oceanogr., 29, 2689-2710.

$\longrightarrow$, and - 2003a: Long extratropical planetary wave propagation in the presence of slowly varying mean flow and bottom topography. Part I: The local problem. J. Phys. Oceanogr., 33, 784-801.

- , and - , 2003b: Long extratropical planetary wave propagation in the presence of slowly varying mean flow and bottom topography. Part II: Ray propagation and comparison with observations. J. Phys. Oceanogr., 33, 802-821.

— D. B. Chelton, and R. de Szoeke, 1997: The speed of observed and theoretical long extratropical planetary waves. $J$. Phys. Oceanogr., 27, 1946-1966.

Liu, Z. Y., 1999: Planetary waves in the thermocline: NonDoppler-shift mode, advective mode and Green mode. Quart. J. Roy. Meteor. Soc., 16, 838-855.

Pedlosky, J., 1996: Ocean Circulation Theory. Springer-Verlag, $453 \mathrm{pp}$.

Rhines, P. B., 1977: The dynamics of unsteady currents. The Sea, E. D. Goldberg, Ed., Marine Modeling, Vol. 6, John Wiley and Sons, 189-318.

Samelson, R. M., 1992: Surface-intensified Rossby waves over rough topography. J. Mar. Res., 50, 367-384.

Schopp, R., 1988: Spinup toward communication between large oceanic subpolar and subtropical gyres. J. Phys. Oceanogr., 18, 1241-1259.

Spydell, M., and P. Cessi, 2003: Baroclinic modes in a two-layer basin. J. Phys. Oceanogr., 33, 610-622.

Sturges, W., and B. G. Hong, 1995: Wind forcing of the Atlantic thermocline along $32^{\circ} \mathrm{N}$ at low frequencies. J. Phys. Oceanogr., 25, 1706-1715.

— - and A. J. Clarke, 1998: Decadal wind forcing of the North Atlantic subtropical gyre. J. Phys. Oceanogr., 28, 659668.

Tailleux, R., 2003: Comments on "The effect of bottom topography on the speed of long extratropical planetary waves." $J$. Phys. Oceanogr., 33, 1536-1541.

—, and J. C. Mc Williams, 2000: Acceleration, creation, and depletion of wind-driven, baroclinic Rossby waves over an ocean ridge. J. Phys. Oceanogr., 30, 2186-2213.

_ and _ 2001: The effect of bottom pressure decoupling on the speed of extratropical, baroclinic Rossby waves. J. Phys. Oceanogr., 31, 1461-1476.

Welander, P., 1970: The thermocline problem. Philos. Trans. Roy. Soc. London, 270A, 415-421.

Young, W. R., and P. B. Rhines, 1982: A theory of the winddriven circulation. Part II: Circulation models with western boundary layer. J. Mar. Res., 40 (Suppl.), 849-872. 